RESIDENT

\& FELLOW

SECTION

Section Editor

Mitchell S.V. Elkind,

MD, MS

M. Reimann, $\mathrm{PhD}$

R. Manz, MD

S. Prieur

H. Reichmann, Prof

T. Ziemssen, MD

Address correspondence and reprint requests to Dr. Manja Reimann, ANF Laboratory, Department of Neurology, University Clinic Carl Gustav Carus, Dresden University of Technology, Fetscherstraße 74, D-01307 Dresden, Germany manjareimann@uniklinikum-dresden.de

\section{Education Research:}

\section{Cognitive performance is preserved in sleep-deprived neurology residents}

Pit

\section{ABSTRACT}

Objective: To test the hypotheses that sleep deprivation in neurology residents is associated with performance deficits and that vigilance and cognitive performance is more compromised after overnight on-call duty compared to night shift.

Methods: Thirty-eight neurology residents of a university teaching hospital participated in a prospective single-blind comparison study. Residents were recruited according to their working schedule and divided into 3 groups: 24 hours overnight on-call duty, night shift, and regular day shift (controls). All participants underwent serial measurements of sleepiness and cognitive performance in the morning directly after or before their shift. Pupillary sleepiness test and Paced Auditory Serial Addition Test were applied. Perceived sleepiness was assessed by a questionnaire.

Results: Sleepiness was increased in residents after night shift and overnight call compared to controls while the type of night duty was not associated with the extent of sleepiness. Sleepdeprived residents did not show any performance deficits on the Paced Auditory Serial Addition Test. Cognitive performance was not associated with sleepiness measures.

Conclusions: Night shift and overnight call duty have a similar impact on alertness in neurology residents. Sleep-deprived neurology residents may be able to overcome sleep loss-related performance difficulties for short periods. Neurology ${ }^{\circledR} 2009 ; 73:$ e99-e103

GLOSSARY

PASAT $=$ Paced Auditory Serial Addition Test; PST = Pupillography Sleepiness Test; PUI = pupillary unrest index; $\mathbf{S S S}=$ self-stated sleepiness.

Despite recent changes in working schedule regulation for clinicians, long working hours remain a common feature in health care. ${ }^{1-3}$ Associated sleep deprivation and fatigue present not only a serious concern for patient safety, but also places the health of health care professionals at risk. Serious problems resulting from sleep loss range from performance deficits and erroneous decision making to increased risk for motor vehicle accidents. ${ }^{2,4}$

The majority of sleepiness studies have been performed in certain medical specialties with a reputation for demanding schedules, such as surgery or intensive care. ${ }^{2}$ Neurologists are frequently underrated in terms of intensity and heaviness of their working schedules due to their mistakenly close relationship to psychiatry. Call rotation on the neurology ward and night shifts at the neurology intensive care unit are as common as in internal medicine or surgery. Actually, neurologists are frequently challenged by the high prevalence of life-threatening strokes in the Western society, where any delay in action or poor performance may be fatal for the patient.

This prompted us to investigate sleepiness and cognitive performance in sleep-deprived and alert neurologists working at a large university neurology clinic. We hypothesized that 1 ) sleep deprivation in neurologists is associated with performance deficits as previously demonstrated for other

From the Autonomic and Neuroendocrinological Laboratory, Department of Neurology (M.R., R.M., S.P., H.R., T.Z.), and Research Group NeuroMetabolism, Department of Neurology and Internal Medicine III (M.R., T.Z.), University Hospital Carl Gustav Carus, Dresden University of Technology, Germany.

Supported by the University Hospital Carl Gustav Carus, Dresden, Germany.

Disclosure: Author disclosures are provided at the end of the article. 
medical specialties and that 2) vigilance as well as cognitive performance is more compromised after 24 hours overnight on-call duty compared to night shift.

METHODS This pilot study included 38 neurology residents (19 women and 19 men, mean age $30 \pm 2$ years) recruited from the Department of Neurology at the University Hospital Carl Gustav Carus, Dresden, Germany. We screened residents for exclusion criteria such as current use of medication known to affect the sleep/ wake cycle or daytime alertness, current psychiatric illness, and sleep disorder diagnosis. We then stratified eligible residents according to their working schedule into 3 groups: group 1, 24 hours overnight on-call duty; group 2, night shift; and group 0 , regular day shift (control).

Definitions of groups. Residents in group 1 (24 hours overnight on-call duty) performed their regular shift from $8 \mathrm{AM}-4$ PM followed by overnight call until 8 AM the next day. During their overnight duty, they were allowed to sleep but there was no scheduled coverage. Residents have usually 3 to 4 overnight calls per month. Residents in group 2 (night shift) worked at the intensive care unit for 7 consecutive days daily from 8 PM to 8 AM the following morning. Afterwards they had 1 week off. Sleeping was not permitted during their shifts. From experience, residents have on average 2 admissions and 30 internal and 3 outpatient consultations per night. Night shift rotation was every 5 to 6 weeks over a period of 1 year. Residents in group 0 (day shift) regularly worked from 7:30 AM to 3:30 PM. However, residents on day shift frequently work overtime.

Standard protocol approvals, registrations, and patient consents. The Ethics Committee on human experimentation of the Dresden University of Technology approved the study and the investigation conformed with the principles outlined in the Declaration of Helsinki. We informed all participating residents about the objectives and procedures of the study and obtained written informed consent prior to their inclusion. The serial data collection was performed at the Autonomic and Neuroendocrinological Laboratory of the University Clinic. We measured all residents before 9 AM directly after their (night) shift rotation or just before day shift commenced (controls). The assistant performing the measurements was blinded. We measured objective sleepiness by Pupillography Sleepiness Test (PST) and cognitive performance by Paced Auditory Serial Addition Test (PASAT). We instructed the residents to abstain from drinking alcoholic beverages, smoking, and drinking coffee for at least 4 hours before the measurements. The residents rated their sleepiness on a 5-point Likert scale based on the statement "Currently I feel." We also recorded the number of hours slept in the previous 24 hours and assessed the perceived recovery effect due to sleep on a 5-point Likert scale. All measurements were serially repeated up to a maximum of 13 times. Measurement frequency, however, varied between individuals according to the rotation schedules $(4.8 \pm 3.3)$.

Pupillary Sleepiness Test. We performed the PST (AMTech, Dossenheim, Germany) in a quiet and darkened room after an initial dark-adapting phase of 15 minutes. During PST, residents wore goggles equipped with infrared light transmitting filter glasses impervious to visible light. They were seated on a comfortable chair and head position was adjusted by a chin rest fixed on a table. An infrared video camera was fixed at a distance of $70 \mathrm{~cm}$ from the examination subject. We instructed the clinicians to maintain fixation on a set of infrared light-emitting diodes. We then recorded spontaneous pupillary oscillations over a period of 11 minutes by infrared video pupillography and evaluated the recording by $25-\mathrm{Hz}$ real-time analysis as published elsewhere. ${ }^{5}$ Pupillary unrest index (PUI) is a measure of pupillomotor hippus in darkness and calculated as an integrated sum of slow movements of the pupillary margin during the measurement period. ${ }^{6}$ This value is usually low in alertness and increases with progressive sleepiness. We also calculated the mean pupil diameter over the entire recording period of 11 minutes. During sleepiness the initial diameter is reduced and the mean pupil size falls below the initial diameter toward the end of the measurement.

Paced Auditory Serial Addition Test. The validated and computer-aided PASAT allows for measuring the capacity and velocity of information processing within the auditory-verbal domain (cognitive performance) ${ }^{7,8}$ The test system entails the subject to continuously add the last 2 numbers of consecutive series and to announce the sum aloud. Numbers from 1 to 9 are announced acoustically in random order by a PC with the screen remaining dark. To avoid practice effects, clinicians were trained on the PASAT at least 3 times before commencing the study. We applied the 60-item short version of the test (maximal score of 60 ). Lower scores (small numbers of correct answers) indicate worse cognitive performance.

Statistical analysis. We used the SPSS software package version 16.0 for Windows (SPSS Inc., Chicago, IL) for all statistical evaluation. Data are presented as median and 25th-75th percentile unless otherwise stated. Owing to the small sample size we assumed non-Gaussian distribution, and hence applied nonparametric tests with Bonferroni correction for comparing groups. Spearman correlation coefficients were calculated. A two-tailed $p<0.05$ was regarded as the level of significance.

RESULTS Before we started the comparison among the 3 groups, we assessed the strength of association between the first measurement and the mean of serial

\begin{tabular}{|c|c|c|c|c|}
\hline \multicolumn{5}{|c|}{ Sleepiness and cognitive performance of neurology residents by type of night duty } \\
\hline Parameters & Overnight call $(n=17)$ & Night shift $(n=6)$ & Control $(n=15)$ & $\chi^{2}\left(p^{*}\right)$ \\
\hline Pupil diameter (mm) & $7.51(6.25-7.75)$ & $7.23(5.18-7.54)$ & $7.21(6.61-8.00)$ & $0.59(0.744)$ \\
\hline Pupillary unrest index (mm/min) & $7.00(4.96-9.44)^{\mathrm{a}}$ & $10.34(7.78-15.07)^{\mathrm{a}}$ & $4.72(3.86-5.09)^{b}$ & $12.88(0.002)$ \\
\hline PASAT score $^{+}$ & $56(49-58)$ & $51(42-58)$ & $54(48-56)$ & $0.67(0.715)$ \\
\hline Self-stated sleepiness ${ }^{\ddagger}$ & $2.3(1.7-2.7)^{a}$ & $2.6(2.2-3.1)^{a}$ & $1.0(0-1.0)^{b}$ & $20.24(<0.001)$ \\
\hline
\end{tabular}

Data are median (interquartile range). Unequal superscript letters indicate significant differences (Mann-Whitney $U$ test). *Kruskal-Wallis test.

+Paced Auditory Serial Addition Test (number of correct answers/60).

*Categorical value. 
average a mean (minimum-maximum) of 2 (1-3) admissions, $2(0-7)$ consultations, and $3(1-5)$ telephone inquiries during overnight on-call duty.

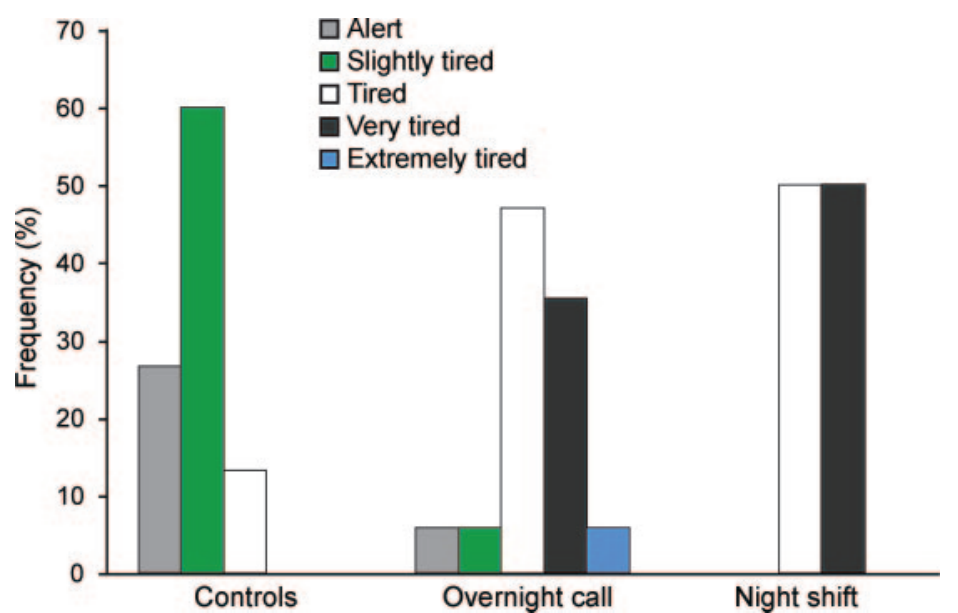

measurements. We revealed significant correlations with $r$ values ranging from 0.763 to 0.904 ( $p<$ $0.001)$. Based on these results, we continued our analysis using the mean values.

PUI and self-stated sleepiness (SSS) were significantly affected by type of night duty while pupil diameter and the PASAT score remained unaffected (table). It appeared that PUI and SSS were significantly higher after the night shift and the 24 hours overnight on-call duty compared to a normal night at home. Residents after night shift and 24 hours overnight on-call duty did not differ with respect to sleepiness measures.

Neurology residents on 24 hours overnight oncall duty had slept on average $4.3(2.8-4.6)$ hours (midshift nap) in the last 24 hours, which was significantly less compared to their colleagues on night shift (5.9 [4.9-7.0] hours, $p=0.006)$ or on day shift (controls) (6.5 [6.0-7.0] hours, $p<0.001)$. The longest sleeping phase during 24 hours overnight oncall duty was $3.0(2.0-3.8)$ hours. Residents had on

\section{Figure 2 Self-stated recovery effect due to sleep in the past 24 hours}

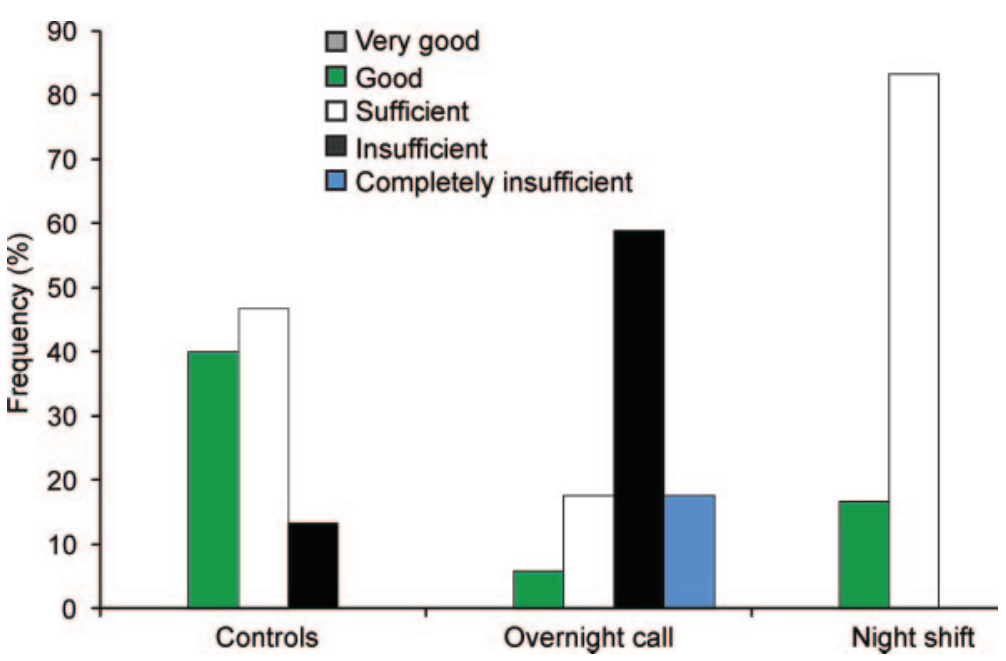

Figure 1 illustrates the proportion of respective responses to the statement "Currently I feel . . ." Figure 2 depicts the self-stated recovery effect due to sleep in the 24 hours preceding the examination.

Correlation analyses did not reveal any association between the PUI and the PASAT score. However, the PUI increased $(r=0.507, p=0.001)$ and the PASAT score decreased $(r=-0.335, p=0.04)$ with increased SSS in the total sample. The perceived level of sleepiness decreased as the number of sleeping hours in the past 24 hours increased $(r=-0.527, p=0.001)$. The above associations could not be confirmed in subgroups $(p>$ $0.05)$.

DISCUSSION Rotating shift work in clinics to provide 24-hour patient care has come increasingly under scrutiny due to negative effects associated with sleep loss, fatigue, and circadian disruption. ${ }^{9,10}$ Although night shift and 24 hours overnight on-call duty considerably differ in terms of number of working hours, permission for midshift naps, and rotation frequency, their effect on sleepiness and cognitive performance has never been distinguished. We hypothesized that residents on 24 hours call rotation would be more affected by sleep loss due to a longer and more irregular working schedule. We investigated this hypothesis in neurology residents of a large university clinic. This specialty group is often underrated in terms of heaviness and intensity of labor and therefore has never been investigated in sleepiness studies. Importantly, previous sleepiness studies in selected medical specialties explicitly emphasized that results must not be extrapolated to other medical specialty groups. ${ }^{3,4,11}$ Although we could not verify the above hypothesis, our results clearly demonstrate that sleepiness is a common problem among neurology residents undergoing night shift and 24 hours overnight on-call duty.

We additionally demonstrated that vigilance measured by PST is in good agreement with SSS. This finding corresponds with previous studies suggesting the PST as a valid and objective tool to detect sleepiness in healthy subjects. ${ }^{6}$

The lack of significant performance decrements in sleep-deprived neurology residents vs controls is intriguing since an association between performance and acute sleep deprivation was found in previous studies. ${ }^{2,4,12}$ Differences in study design, medical specialty, and methods for vigilance testing limit comparisons across studies. However, one study also failed to show a significant performance decrement on the complex PASAT test in sleep-deprived normal subjects. ${ }^{13}$ The investigators concluded that university-based research may func- 
tion as a motivational incentive, which tends to offset sleepiness effects on performance. ${ }^{13}$

Alternatively, the applied method for performance testing may have been suboptimal for our population since its clinical utility is mainly proven in neuropsychological syndromes. However, more recent studies also employed the PASAT in healthy adults with satisfactory results. ${ }^{7,14}$ Originally assumed to measure rate of information processing, the PASAT is now recognized as tapping into different types of cognitive processes. ${ }^{15}$ This multifactorial nature may complicate the interpretation of test result from sleep-deprived subjects especially under the assumption that sleep loss affects different cognitive pathways differentially. The consistently high PASAT score across all study groups suggests that sleep-deprived neurology residents are able to overcome performance difficulties for a limited time period. This assumption seems plausible since stressful working situations are routine in the clinic and thus may facilitate partial conditioning. Although there is evidence that sleep-deprived resident doctors are prone to committing medical errors, ${ }^{4,9}$ tasks of short duration may be less likely to detect performance deficits in chronically sleep deprived individuals. ${ }^{2}$

Importantly, we adequately controlled for practice effects ${ }^{8}$ first by using a control group and secondly by training the clinicians on the PASAT prior to the study. Consequently, we obtained a very good agreement between the first measurement and the mean of serial measurements. In addition, major confounders of the PASAT such as age, gender, education, and ethnicity ${ }^{14,16}$ were accounted for by creating a homogenous study sample.

Nevertheless, it must be considered that our results are compromised by a questionable rested control group. Correspondingly, the average amount of sleep in our controls was equivalent to the accepted core sleep requirement of 6.5 hours (many were also below). ${ }^{17}$ In addition, nearly two thirds of the controls felt fairly to poorly rested, which indicates chronic partial sleep deprivation (figure 3). This finding is relevant as chronic partial sleep deprivation appears to be cumulative with respect to performance decrements. ${ }^{18}$

Neurology residents on night shift and overnight call are affected to a similar extent by sleepiness. Increased sleepiness, however, did not affect performance on the complex PASAT test. It seems that sleepdeprived neurology residents may be able to overcome sleep loss-related performance difficulties for short periods. This, however, may not necessarily apply for more demanding procedures outside the laboratory.

\section{AUTHOR CONTRIBUTIONS}

Statistical analysis was conducted by Dr. Manja Reimann.

\section{ACKNOWLEDGMENT}

The authors thank all neurology residents for their participation in this study.

\section{DISCLOSURE}

Dr. Reimann, Dr. Manz, and S. Prieur report no disclosures. Dr. Reichmann serves on scientific advisory boards, receives speaker honoraria, and/or receives funding for travel from Cephalon, Inc., Novartis, Teva Pharmaceutical Industries Ltd., Lundbeck Inc., GlaxoSmithKline, Boehringer Ingelheim, Bayer Schering Pharma., UCB/Schwarz Pharma, Desitin Pharmaceuticals, GmbH, Pfizer Inc., and Solvay Pharmaceuticals, Inc. Dr. Ziemssen has received speaker honoraria from Biogen Idec, SanofiAventis, Merck Serono, Novartis, Teva Pharmaceutical Industries Ltd., and Bayer Schering Pharma; serves as a consultant for Teva Pharmaceutical Industries Ltd., Novartis, and Bayer Schering Pharma; and receives research support from the Roland Ernst Foundation.

\section{REFERENCES}

1. Reddy R, Guntupalli K, Alapat P, Surani S, Casturi L, Subramanian S. Sleepiness in medical ICU residents. Chest 2009;135:81-85.

2. Veasey S, Rosen R, Barzansky B, Rosen I, Owens J. Sleep loss and fatigue in residency training: a reappraisal. JAMA 2002;288:1116-1124.

3. Surani S, Subramanian S, Aguillar R, Ahmed M, Varon J. Sleepiness in medical residents: impact of mandated reduction in work hours. Sleep Med 2007;8:90-93.

4. Arnedt JT, Owens J, Crouch M, Stahl J, Carskadon MA. Neurobehavioral performance of residents after heavy night call vs after alcohol ingestion. JAMA 2005;294:1025-1033.

5. Ludtke H, Wilhelm B, Adler M, Schaeffel F, Wilhelm H. Mathematical procedures in data recording and processing of pupillary fatigue waves. Vision Res 1998;38:2889-2896.

6. Wilhelm B, Wilhelm H, Ludtke H, Streicher P, Adler M. Pupillographic assessment of sleepiness in sleep-deprived healthy subjects. Sleep 1998;21:258-265.

7. Diehr MC, Cherner M, Wolfson TJ, Miller SW, Grant I, Heaton RK. The 50 and 100-item short forms of the Paced Auditory Serial Addition Task (PASAT): demographically corrected norms and comparisons with the full PASAT in normal and clinical samples. J Clin Exp Neuropsychol 2003;25:571-585.

8. Tombaugh TN. A comprehensive review of the Paced Auditory Serial Addition Test (PASAT). Arch Clin Neuropsychol 2006;21:53-76.

9. Barger LK, Cade BE, Ayas NT, et al. Extended work shifts and the risk of motor vehicle crashes among interns. N Engl J Med 2005;352:125-134.

10. Gold DR, Rogacz S, Bock N, et al. Rotating shift work, sleep, and accidents related to sleepiness in hospital nurses. Am J Public Health 1992;82:1011-1014.

11. Saxena AD, George CF. Sleep and motor performance in oncall internal medicine residents. Sleep 2005;28:1386-1391.

12. Grantcharov TP, Bardram L, Funch-Jensen P, Rosenberg J. Laparoscopic performance after one night on call in a surgical department: prospective study. BMJ 2001;323: 1222-1223.

13. Hood B, Bruck D. A comparison of sleep deprivation and narcolepsy in terms of complex cognitive performance and subjective sleepiness. Sleep Med 2002;3:259-266.

14. Diehr MC, Heaton RK, Miller W, Grant I. The Paced Auditory Serial Addition Task (PASAT): norms for age, education, and ethnicity. Assessment 1998;5:375-387.

15. Madigan NK, DeLuca J, Diamond BJ, Tramontano G, Averill A. Speed of information processing in traumatic 
brain injury: modality-specific factors. J Head Trauma Rehabil 2000;15:943-956.

16. Wills $S$, Leathem J. The effects of test anxiety, age, intelligence level, and arithmetic ability on Paced Auditory Serial Addition Test performance. Appl Neuropsychol 2004;11: $180-187$.
17. Bonnet $\mathrm{MH}$, Arand DL. We are chronically sleep deprived. Sleep 1995;18:908-911.

18. Dinges DF, Pack F, Williams K, et al. Cumulative sleepiness, mood disturbance, and psychomotor vigilance performance decrements during a week of sleep restricted to 4-5 hours per night. Sleep 1997;20:267-277. 


\section{Neurology}

\section{Education Research: Cognitive performance is preserved in sleep-deprived neurology residents}

M. Reimann, R. Manz, S. Prieur, et al. Neurology 2009;73;e99-e103

DOI 10.1212/WNL.0b013e3181c34a93

\section{This information is current as of November 23, 2009}

\section{Updated Information \& Services}

References

Citations

Subspecialty Collections

Permissions \& Licensing

Reprints including high resolution figures, can be found at: http://n.neurology.org/content/73/21/e99.full

This article cites 18 articles, 1 of which you can access for free at: http://n.neurology.org/content/73/21/e99.full\#ref-list-1

This article has been cited by 1 HighWire-hosted articles: http://n.neurology.org/content/73/21/e99.full\#\#otherarticles

This article, along with others on similar topics, appears in the following collection(s):

\section{Attention}

http://n.neurology.org/cgi/collection/attention

Executive function

http://n.neurology.org/cgi/collection/executive_function

Medical care

http://n.neurology.org/cgi/collection/medical_care

Patient safety

http://n.neurology.org/cgi/collection/patient_safety

Professional conduct and ethics

http://n.neurology.org/cgi/collection/professional_conduct_and_ethics

Information about reproducing this article in parts (figures,tables) or in its entirety can be found online at:

http://www.neurology.org/about/about_the_journal\#permissions

Information about ordering reprints can be found online:

http://n.neurology.org/subscribers/advertise

Neurology ${ }^{\circledR}$ is the official journal of the American Academy of Neurology. Published continuously since 1951, it is now a weekly with 48 issues per year. Copyright . All rights reserved. Print ISSN: 0028-3878. Online ISSN: 1526-632X.

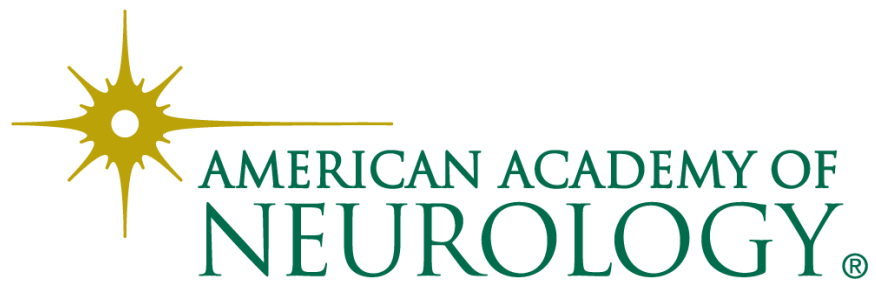

